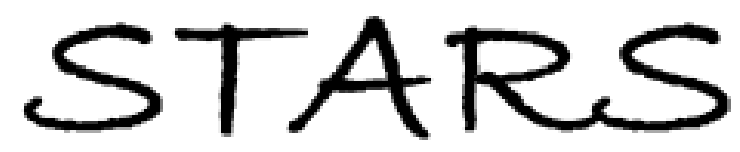

University of Central Florida

STARS

$1-1-2013$

\title{
Theory of radiative electron attachment to molecules: Benchmark study of $\mathrm{CN}$
}

Nicolas Douguet

Samantha Fonseca dos Santos

Maurice Raoult

Olivier Dulieu

Ann E. Orel

See next page for additional authors

Find similar works at: https://stars.library.ucf.edu/facultybib2010

University of Central Florida Libraries http://library.ucf.edu

This Article is brought to you for free and open access by the Faculty Bibliography at STARS. It has been accepted for inclusion in Faculty Bibliography 2010 s by an authorized administrator of STARS. For more information, please contactSTARS@ucf.edu.

\section{Recommended Citation}

Douguet, Nicolas; Fonseca dos Santos, Samantha; Raoult, Maurice; Dulieu, Olivier; Orel, Ann E.; and Kokoouline, Viatcheslav, "Theory of radiative electron attachment to molecules: Benchmark study of CN" (2013). Faculty Bibliography 2010s. 3906.

https://stars.library.ucf.edu/facultybib2010/3906

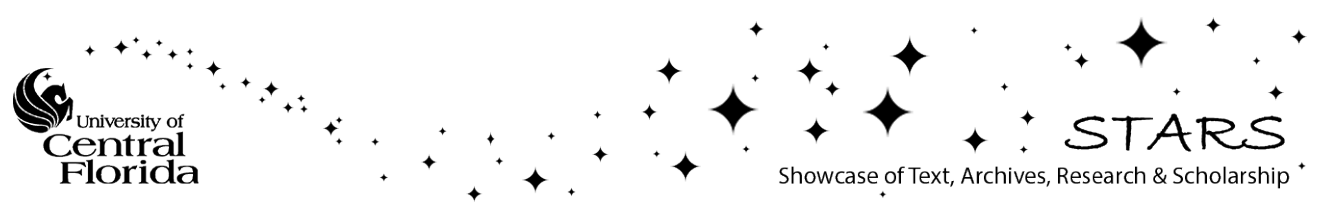




\section{Authors}

Nicolas Douguet, Samantha Fonseca dos Santos, Maurice Raoult, Olivier Dulieu, Ann E. Orel, and Viatcheslav Kokoouline 


\title{
乌s \\ Theory of radiative electron attachment to molecules: Benchmark study of $\mathrm{CN}^{-}$
}

\author{
Nicolas Douguet, ${ }^{1}$ Samantha Fonseca dos Santos, ${ }^{1}$ Maurice Raoult, ${ }^{2}$ Olivier Dulieu, ${ }^{2}$ \\ Ann E. Orel, ${ }^{1}$ and Viatcheslav Kokoouline ${ }^{3}$ \\ ${ }^{1}$ Department of Chemical Engineering and Materials Science, University of California, Davis, California 95616, USA \\ ${ }^{2}$ Laboratoire Aimé Cotton, CNRS/Université Paris-Sud/ENS-Cachan, Bât. 505, Campus d'Orsay, 91405 Orsay Cedex, France \\ ${ }^{3}$ Department of Physics, University of Central Florida, Orlando, Florida 32816, USA \\ (Received 18 September 2013; published 25 November 2013; corrected 3 December 2013)
}

\begin{abstract}
We have developed an approach based on first principles to study the process of radiative electron attachment (REA) to linear molecules of astrophysical interest in collisions between the molecules and electrons at energies below $1 \mathrm{eV}$. The approach is based on accurate ab initio calculations of electronic bound and continuum states of the negative ion. The electronic continuum states are obtained with the complex-Kohn variational method. The benchmark calculation for the REA to the simplest negative ion $\mathrm{CN}^{-}$, which was recently observed in the interstellar medium, has produced a relatively low rate coefficient $\alpha(T)=7.3 \times 10^{-16} \mathrm{~cm}^{3} / \mathrm{s}$ at $T=30 \mathrm{~K}$. Moreover, our results are shown to agree well with microscopic reversibility applied on a recent photodetachment experiment on $\mathrm{CN}^{-}$. Finally, the study confirms a previous assessment that the $\mathrm{CN}^{-}$ion is unlikely be formed by REA in the interstellar medium.
\end{abstract}

DOI: 10.1103/PhysRevA.88.052710

PACS number(s): 34.80.Bm, 98.38.Dq, 95.30.Ft, 33.80.-b

\section{INTRODUCTION}

More than 30 years ago, Herbst suggested that negative molecular ions could be formed in the interstellar medium (ISM) through the process of radiative attachment of a lowenergy electron to a neutral radical [1]. The existence of anions in the ISM has been recently confirmed with the discovery of several carbon chain negative molecular ions in space, $\mathrm{C}_{6} \mathrm{H}^{-}$ [2-6], $\mathrm{C}_{4} \mathrm{H}^{-}$[7], $\mathrm{C}_{8} \mathrm{H}^{-}$[8], $\mathrm{C}_{3} \mathrm{~N}^{-}$[9], $\mathrm{C}_{5} \mathrm{~N}^{-}$[3,6], and $\mathrm{CN}^{-}$ [10]. The smallest discovered negative ion $\mathrm{CN}^{-}$was observed for the first time in 2006 by M. Agúndez et al. [10] in the Cstar envelope IRC + 10216 via microwave spectroscopy. The formation of the latter diatomic ion through radiative electronic attachment (REA) is the main focus of the present study.

Using a simple statistical approach, known as phase-space theory (PST), Herbst and coworkers have calculated the temperature-dependent rate coefficient $\alpha(T)$ for formation of several negative ions via REA, including most of the ions listed above. We use symbols $\mathrm{M}$ and $\mathrm{M}^{-}$for a particular molecular radical and its counterpart negative ion, respectively. Assuming that the major mechanism for formation and destruction of $\mathrm{M}^{-}$are REA and mutual neutralization with positive ions, one can deduce the abundance ratio $\left[\mathrm{M}^{-}\right] /[\mathrm{M}]$ in a specific medium at a thermal equilibrium from the rate coefficients $\alpha(T)$, the temperature and the density of free electrons and positive ions in the medium. The predictions of the PST for these ratios are in relatively good agreement with the astrophysical observations for $\mathrm{C}_{8} \mathrm{H}^{-}, \mathrm{C}_{6} \mathrm{H}^{-}, \mathrm{C}_{5} \mathrm{~N}^{-}$, and $\mathrm{C}_{3} \mathrm{~N}^{-}$ions, but disagree for $\mathrm{C}_{4} \mathrm{H}^{-}$ and $\mathrm{CN}^{-}$. Indeed, the calculated rate coefficient for REA to $\mathrm{CN}$ is about $10^{-17} \mathrm{~cm}^{3} / \mathrm{s}$ at $10 \mathrm{~K}$ [11], which is some 6-7 orders of magnitude lower than the value needed to explain the $\left[\mathrm{CN}^{-}\right] /[\mathrm{CN}]$ ratio obtained from the astrophysical observations. On the other hand, in spite of the extremely low REA rate coefficient obtained theoretically for $\mathrm{CN}$, it is still believed that REA should be the primary mechanism for formation of the larger carbon-chain molecular ions. This implies that the REA rate for these ions should be some 5-7 orders of magnitude larger than the predicted REA rate in $\mathrm{CN}^{-}$formation.

In view of the uncertainty of the formation pathway for negative ions, we propose to apply a fully quantum-mechanical model of REA to a neutral radical, using first principles only, thus refining the PST by going beyond a statistical approach. One goal of this study is to obtain a precise and reliable REA rate for $\mathrm{CN}^{-}$formation and determine if REA is an efficient process in $\mathrm{CN}^{-}$formation. Beyond the particular example of $\mathrm{CN}$, our main motivation is to present a complete quantum-mechanical REA theory, employing a minimum of approximations. To our knowledge, only classical or statistical approximate models have been reported so far in the literature, such that the present work represents a benchmark study, applied to a system with only a few degrees of freedom, such that the electronic continuum, the vibrational and rotational motion of the molecule, and the process of radiative emission, can all be treated at once. The theory and the conclusions suggested in this work will serve as a route towards studying the formation of larger molecular ions by REA, which represents a natural extension of the presented approach on $\mathrm{CN}$.

The paper is organized as follow. In the next section, we discuss the state of the art on REA, describing briefly the widely used phase-space theory, pointing out its strengths and limitations, and proposing some improvements. In Sec. III, we develop the theoretical approach and present the REA crosssection formula, whose derivation represents the main theoretical result of the present study and is presented in the Appendix to improve the clarity of the text. Section IV describes our $a b$ initio calculations and presents potential curves, as well as the molecular frame transition dipole moment (TDM) values from a continuum electron to the final ground state of $\mathrm{CN}^{-}$, obtained via the complex Kohn variational method. The obtained numerical results are presented in Sec. V. The last section is devoted to our conclusions, where we discuss other possible pathways for $\mathrm{CN}$ formation and the applicability of the present REA model for larger molecular radicals.

\section{STATE OF THE ART AND DISCUSSION}

The statistical PST is the most accepted theory of REA used to model formation of negative molecular ions in the interstellar space [1,4,11-15]. The PST assumes that an 
incoming electron will always attach to the radical, with the corresponding attachment cross section given by $\pi / k^{2}$ for any momentum $\vec{p}=\hbar \vec{k}$ of the incident electron, forming a temporary state with a finite lifetime [4]. Once the electron is attached to the radical, the extra energy of the compound can be released via two different competing processes. The first outcome is the autodetachment of the electron from the radical. In PST, the statistical rate coefficient for autodetachment is inversely proportional to the density of states $\rho$ at the total energy of the molecular ion, such that autodetachment should decrease significantly for ions with many degrees of freedom. The second process for energy release, which leads to negative ion stabilization, is radiative relaxation. The ratio between the rate of radiative relaxation and autodetachment represents the key parameter in the PST to determine the total rate of the REA reaction.

Therefore, the PST has a great advantage of providing rates for formation of large negative ions by REA, with only few parameters needed. On the other hand, PST employs some approximations that must be critically revised. Certainly the strongest approximation used in PST is the assumption of $100 \%$ efficiency in electronic capture. Without performing detailed quantum scattering calculations, the relevance of such an approximation is relatively hard to quantify and might overestimate considerably the actual attachment probability. Indeed, there are only three different ways how the electron could attach to the radical:

(i) The first possibility demands the existence of a broad shape resonance located at electronic energy just above detachment threshold. In such a case, a low-energy electron can be trapped efficiently in the inner-core region into a temporary state, which could then stabilize by radiative emission. However, the existence of such low-lying shape resonances is not guaranteed and, most likely, such states do not exist for all discovered negative ions. In fact, Harrison et al. have performed electron scattering calculations for $\mathrm{CN}$ [16] and $\mathrm{C}_{3} \mathrm{~N}$ [17] radicals and found no low-lying electronic resonances for these systems. Even if such resonances were to exist in other negative ions, the capture probability could still be significantly smaller than unity.

(ii) The second possibility for attachment, suggested by Herbst and coworkers, is such that the electron is directly captured in an excited vibrational resonance attached to the electronic ground state of the negative ion. For $\mathrm{CN}^{-}$, such a vibrational level with the energy high enough for the electron detachment, would be $v=18$ or higher (see Fig. 1, which shows energies of two excited vibrational states of $\mathrm{CN}^{-}, v^{-}=18$ and 19 ). The capture is mediated by a nonBorn-Oppenheimer interaction between the initial electronic continuum state of $e^{-}+\mathrm{M}$ and a final rovibrationally excited state of $\mathrm{M}^{-}$in its electronic ground state. Because the affinity of these radicals is large $(\sim 3-5 \mathrm{eV})$, the non-BornOppenheimer couplings between these electronic states, well separated in energy, are expected to be small. In addition, the excited vibrational state of the formed $\mathrm{M}+e^{-}$resonance state has generally a small overlap with the initial vibrational level of the target molecule. For example, for $\mathrm{CN}$, the vibrational factor $\left|\left\langle v_{f}^{-} \mid \frac{\partial}{\partial R} v_{i}\right\rangle\right|^{2}$ between the initial vibrational level $v_{i}=0$ of $\mathrm{CN}$ and the final level $v_{f}^{-}=19$ of $\mathrm{CN}^{-}$in the non-

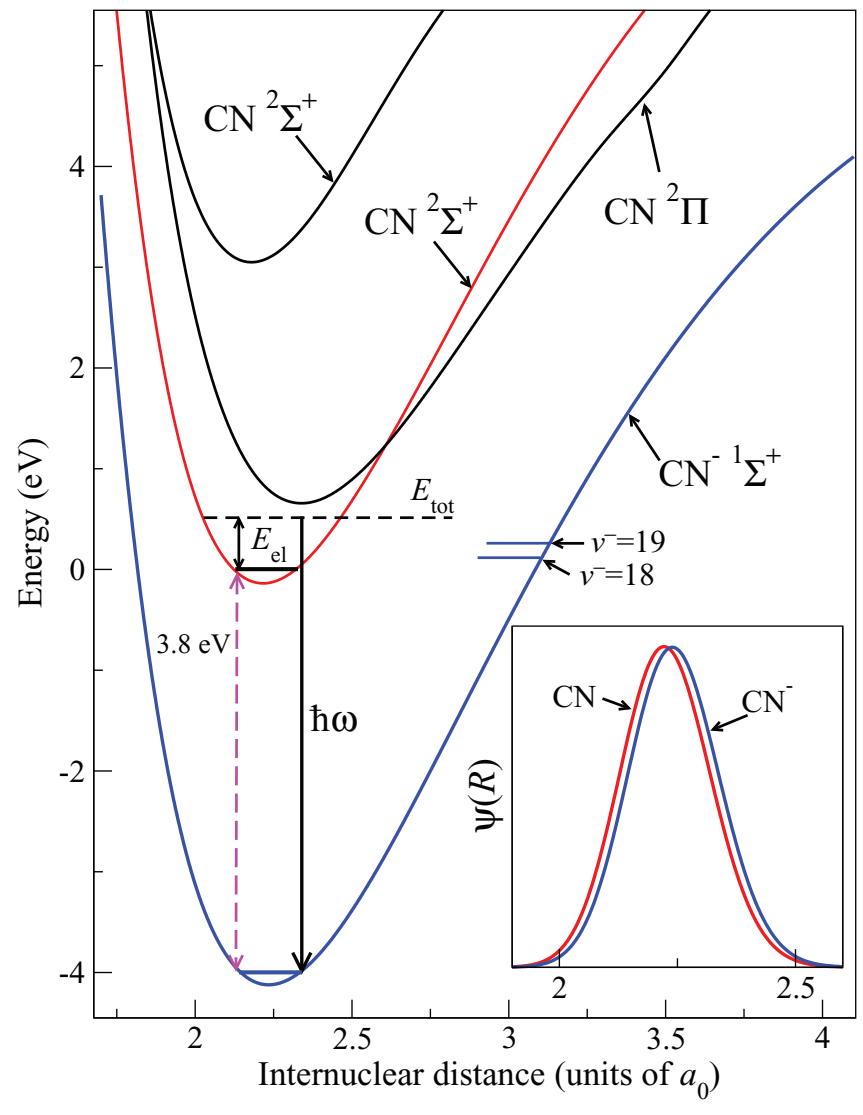

FIG. 1. (Color online) This figure demonstrates the REA process and shows potential energy curves of the $\mathrm{CN}^{-1} \Sigma^{+}$electronic state and several lowest electronic doublet states of $\mathrm{CN}$. In the interstellar medium, REA starts with $\mathrm{CN}$ in its ground vibronic state. During the electron-molecule collision, the $\mathrm{CN}+e^{-}$complex with energy $E_{\text {tot }}$ can emit a photon of energy $\hbar \omega$ bringing the complex into a stable rovibrational state of $\mathrm{CN}^{-}$as shown in the figure. Because the potential curves of the $\mathrm{CN}^{-1} \Sigma^{+}$state and the lowest $\mathrm{CN}$ state are nearly parallel, the vibrational wave functions of the states are almost identical (see the inset). Since the $\mathrm{CN}$ and $\mathrm{CN}^{-}$molecules have almost identical shapes in the electronic potential and because of the weak dependence of dipole moments on $R$ (see Fig. 4), when a photon is emitted, the vibrational quantum number is almost conserved. The origin of energy is taken at the ground vibrational level of $\mathrm{CN}$.

Born-Oppenheimer coupling term is $3.6 \times 10^{-9} a_{0}^{-1}$. A more detailed analysis for $\mathrm{CN}$ shows that the upper bound for the cross section for the capture of the electron into $v_{f}^{-}=19$ is of the order of $10^{-12} / E_{e l}$ in units of $a_{0}^{2}$, where $E_{e l}$ is the incident electron energy measured in units of Hartree. At energy $E_{e l}=10^{-4}$ Hartree or $2.7 \mathrm{meV}$, it gives $2.5 \times 10^{-25} \mathrm{~cm}^{2}$, which is about three orders of magnitude smaller than the cross section from process (i) considered in this study (see Fig. 5).

(iii) Finally, another way to attach an electron to $M$ is through weakly bound dipole states of the electron in $\mathrm{M}$. Such states are situated just below the detachment threshold and can only exist if the permanent dipole moment of the radical is supercritical, which is the case for large carbon chain molecules such as $\mathrm{C}_{3} \mathrm{~N}$ [17], but not for $\mathrm{CN}$. When dipole states 
exist, the Franck-Condon overlap with rovibrational levels of such dipole electronic states is close to unity and the non-BornOppenheimer coupling between the electronic-continuum and dipole state could thus be strong, making the electron capture much more efficient than the capture into the electronic ground state of $\mathrm{M}^{-}$. This process is similar to the indirect dissociative recombination mechanism in positive molecular ions [18], for which the electron is captured in excited Rydberg states. However, in contrast to the case of Rydberg states, no lower accessible electronic states exist once the electron is attached to a dipole state (in the case of Rydberg states, the electron energy is gradually reduced, after the electron is trapped by the molecular ion, through transitions to lower Rydberg states and excitation at the same time of rotational or/and vibrational degrees of freedom of the molecule). Therefore, there is no pathway for stabilization of the $\mathrm{M}+e^{-}$system other than a spontaneous emission to the ground electronic (nondipolar) state of $\mathrm{M}^{-}$. The corresponding radiative lifetime is very long, because the electronic orbit in a dipole state has a very large radius in comparison with the size of the core ground electronic state of the negative ion; or in other words, the electronic TDM is very low between the dipolar and ground electronic states of $\mathrm{M}^{-}$. This type of process must be explored in future studies on negative ions supporting dipole states.

In our treatment, we will not consider process (iii) because $\mathrm{CN}$ has no dipole states. We also discard process (ii), because its contribution to the total REA cross section is significantly smaller compared to process (i). Thus, we only consider process (i). As will be explained in Sec. III, the general idea proposed by Herbst that the electron is initially captured by an intermediate state and stabilized by radiative stabilization, as described by process (i), is incorporated in the present fully quantum approach if such an intermediate state does exist, but the REA process in the present treatment is not separated here into several steps: We consider spontaneous emission of a photon from an electron-molecule scattering state to any possible rovibrational state of the negative ion in its stable electronic bound state. If a shape resonance were to exist, the probability density of the electron would increase at short distances from the core and would dramatically enhance the probability of spontaneous emission.

\section{THEORY OF RADIATIVE ELECTRON ATTACHMENT}

We assume that the initial state of the neutral molecule $\mathrm{CN}$ having total energy $E_{i}$ is a given electronic Born-Oppenheimer wave function $\omega_{\Gamma}$, where the index $\Gamma$ refers to different electronic target channels, a given rotational state specified by the angular momentum $j$ and its projection $\mu$ in the molecular frame (MF) coordinate, and a vibrational wave function $\chi_{v}^{j}$ of CN. The wave function $\chi_{v}^{j}$ is calculated using the Born-Oppenheimer potential modified by the centrifugal term $\left[j(j+1)-\mu^{2}\right] / 2 \mu_{C N} R^{2}$, where $R$ is the internuclear coordinate and $\mu_{C N}$ is the reduced mass of $\mathrm{CN}$. The initial state of the incident electron is the plane wave $e^{i k \tilde{z}}$, where $\tilde{z}$ determines the direction in the laboratory frame (LF) of the incident electron momentum having energy $E_{e l}=$ $(\hbar k)^{2} /\left(2 m_{e}\right)$, where $m_{e}$ is the mass of the electron.

The final state of the process, with energy $E_{f}$, is a bound state of the negative ion $\mathrm{CN}^{-}$specified by its electronic wave function $\Psi_{f}$, the $\mathrm{CN}^{-}$angular momentum $J_{f}$ and its projection $\Lambda_{f}$ in $\mathrm{MF}$, and by the vibrational wave function $\chi_{v_{f}}^{J_{f}}$ of $\mathrm{CN}^{-}$. In the present study, the initial electronic state of $\mathrm{CN}$ is $\mathrm{X}^{2} \Sigma^{+}$and the final electronic state of $\mathrm{CN}^{-}$is always $X^{1} \Sigma^{+}$. The process of REA considered in our approach is described in Fig. 1.

The REA cross section averaged over initial orientations of $\mathrm{CN}$ in space and summed over possible final orientations of $\mathrm{CN}^{-}$, with final angular momentum $J_{f}$, is given in Eq. (1)

$$
\sigma_{f i}=\frac{4}{3} \frac{\pi \omega^{3} m_{e}}{k^{2} \hbar^{2} c^{3}} \sum_{J l}\left|\sum_{\lambda \pi} C_{J \Lambda 1 \pi}^{J_{f} \Lambda_{f}} C_{l \lambda j \mu}^{J \Lambda} d_{\pi, \Gamma l \lambda}^{\left(v \rightarrow v_{f}\right)}\right|^{2} .
$$

The above formula represents the key result of the present theory and its derivation is given in the Appendix. In this formula, $\omega$ is the frequency of the emitted photon, $\hbar \omega=$ $E_{i}+E_{e l}-E_{f} ; d_{\pi, \Gamma l \lambda}^{\left(v \rightarrow v_{f}\right)}$ are the components of the transition dipole moment operator, with $\pi=-1,0,+1$, between the initial electronic wave function $\Psi_{\Gamma l \lambda}$ of the $\mathrm{CN}+e^{-}$system, and the final electronic wave function $\Psi_{f}$ of $\mathrm{CN}^{-}$, integrated over the initial $\chi_{v}^{j}(R)$ and final $\chi_{v_{f}}^{J_{f}}(R)$ vibrational wave functions of $\mathrm{CN}$ and $\mathrm{CN}^{-}$

$$
d_{\pi, \Gamma l \lambda}^{\left(v \rightarrow v_{f}\right)}=\int \chi_{v_{f}}^{J_{f}}(R)\left\langle\Psi_{f}\left|d_{\pi}\right| \Psi_{\Gamma l \lambda}\right\rangle_{r} \chi_{v}^{j}(R) d R
$$

(see details in the Appendix); $J$ is the initial total angular momentum, $l$ is the electronic partial wave angular momentum, and $\lambda$ its projection in the MF. Finally, $C_{J \Lambda 1 \pi}^{J_{f} \Lambda_{f}}$ and $C_{l \lambda j \mu}^{J \Lambda}$ are the Clebsch-Gordan coefficients [19] and $d_{\pi}$ is the electronic dipole moment operator.

For the case of the $\mathrm{CN}$ molecule in its electronic ground state $X^{2} \Sigma^{+}$, the formula significantly simplifies once the sum over all final angular momenta $J_{f}$ is evaluated. As shown in the Appendix, the REA cross section for transition to any possible rotational state of $\mathrm{CN}^{-}$is then given by

$$
\sigma_{i}=\frac{4}{3} \frac{\pi \omega^{3} m_{e}}{k^{2} \hbar^{2} c^{3}} \sum_{l \pi}\left|d_{\pi, \Gamma l-\pi}^{\left(v \rightarrow v_{f}\right)}\right|^{2} .
$$

In the above formula, the index $\lambda$ is replaced by $-\pi$, since for the dipole transitions $\mathrm{CN}\left({ }^{2} \Sigma^{+}\right) \rightarrow \mathrm{CN}^{-}\left({ }^{1} \Sigma^{+}\right)$, we have $\lambda=-\pi$.

Therefore, if one neglects the difference in energy due to the rotation of $\mathrm{CN}$, the final cross section summed over all possible final states is independent on the initial rotational state $j$ of the $\mathrm{CN}$ molecule.

\section{STRUCTURE AND SCATTERING CALCULATIONS}

The main configuration of the initial $X^{2} \Sigma^{+}$electronic ground state of $\mathrm{CN}$ is $1 \sigma^{2} 2 \sigma^{2} 3 \sigma^{2} 4 \sigma^{2} 1 \pi^{4} 5 \sigma^{1}$. Following the REA reaction, the final stable closed shell $X^{1} \Sigma^{+}$state of the negative ion $\mathrm{CN}^{-}$is formed with main configuration $1 \sigma^{2} 2 \sigma^{2} 3 \sigma^{2} 4 \sigma^{2} 1 \pi^{4} 5 \sigma^{2}$. We have determined properties of the target and negative ion ground state, by performing $a b$ initio calculations with the MOLPRO suite [20]. Using the aug-cc-pVQZ basis for the nitrogen and carbon, we performed a MRCI calculation with all double excitations and obtained accurate potential energy curves, presented for the first three states $X^{2} \Sigma^{+}, A^{2} \Pi^{+}$, and $B^{2} \Sigma^{+}$of $C N$, and the $X^{1} \Sigma^{+}$ground state of $\mathrm{CN}^{-}$(see Fig. 1). The calculations have reproduced 
several characteristics of $\mathrm{CN}$ reported previously by other authors. We have obtained a value of $3.80 \mathrm{eV}$ for the zero-point energy affinity, which is in good agreement with the experimental measurement of $3.86 \mathrm{eV}$ [21]. The calculated vibrational frequency of $2057 \mathrm{~cm}^{-1}$ is also in good agreement with previous theoretical studies giving $2207 \mathrm{~cm}^{-1}$ [22], $2057 \mathrm{~cm}^{-1}$ [23], and an experimental measurement of $2068.6 \mathrm{~cm}^{-1}$ [24].

In order to determine the REA cross section in the dipole approximation for radiative processes, the following matrix elements of the fixed-nuclei TDM are needed:

$$
\begin{aligned}
d_{\pi, \Gamma l \lambda} \equiv & \left\langle\Psi_{f}\left|d_{\pi}\right| \Psi_{\Gamma l \lambda}\right\rangle \\
= & -\sum_{k=1}^{N} \int \Psi_{f}^{*}\left(r_{1}, \ldots, r_{N}\right) e r_{k \pi} \Psi_{\Gamma l \lambda}\left(r_{1}, \ldots, r_{N}\right) \\
& \times d^{3} r_{1}, \ldots, d^{3} r_{N},
\end{aligned}
$$

where $N$ is the number of electrons, $r_{k}$ represents the coordinates of the $k$ th electron, and $r_{k \pi}$ is one of the three cyclic components $(\pi=0, \pm 1)$ of the coordinate of the $k$ th electron

$$
r_{k \pi}= \begin{cases}z_{k}, & \pi=0 \\ \mp\left(x_{k} \pm i y_{k}\right) / \sqrt{2}, & \pi= \pm 1 .\end{cases}
$$

We use the complex Kohn variational method [25,26] to describe the $e^{-}-\mathrm{CN}$ scattering. In the method, the wave function representing the electron-neutral scattering, with the neutral target in the electronic state $\omega_{\Gamma}$, is expressed as

$$
\Psi_{\Gamma l \lambda}=\sum_{\Gamma^{\prime}} \hat{\mathcal{A}}\left(\omega_{\Gamma^{\prime}} F_{\Gamma^{\prime} \Gamma}^{+}\right)+\sum_{j} b_{j}^{\Gamma} \Theta_{j},
$$

where the first sum runs over energetically open neutral states described by $(N-1)$-electron wave functions $\omega_{\Gamma}$ and the second sum runs over $N$-electron configuration-state functions $\Theta_{j}$ representing penetration and correlation terms. The operator $\hat{\mathcal{A}}$ antisymmetrizes the wave function with respect to electron permutation. The functions $F_{\Gamma^{\prime} \Gamma}^{+}$are expanded as:

$$
\begin{aligned}
F_{\Gamma^{\prime} \Gamma}^{+}= & \sum_{j^{\prime}} c_{j^{\prime}}^{\Gamma^{\prime} \Gamma} \phi_{j^{\prime}}(r)+\sum_{l^{\prime} \lambda^{\prime}}\left[j_{l^{\prime}}\left(k_{\Gamma} r\right) \delta_{l^{\prime} l} \delta_{\lambda^{\prime} \lambda} \delta_{\Gamma^{\prime} \Gamma}\right. \\
& \left.+T_{l^{\prime} \lambda^{\prime} ; l \lambda}^{\Gamma^{\prime} \Gamma} h_{l^{\prime}}^{+}\left(k_{\Gamma} r\right)\right] Y_{l^{\prime} \lambda^{\prime}}(\hat{r}) / r,
\end{aligned}
$$

where $\phi_{j^{\prime}}$ is a set of square integrable (Cartesian-Gaussian) functions and $j_{l^{\prime}}$ and $h_{l^{\prime}}^{+}$are partial-wave continuum radial functions, behaving asymptotically as

$$
\begin{array}{r}
j_{l^{\prime}}\left(k_{\Gamma^{\prime}} r\right) \rightarrow \frac{1}{\sqrt{k_{\Gamma^{\prime}}}} \sin \left(k_{\Gamma^{\prime}} r-\frac{l^{\prime} \pi}{2}\right), \\
h_{l^{\prime}}^{+}\left(k_{\Gamma^{\prime}} r\right) \rightarrow \frac{1}{\sqrt{k_{\Gamma^{\prime}}}} \exp \left(i\left[k_{\Gamma^{\prime}} r-\frac{l^{\prime} \pi}{2}\right]\right) .
\end{array}
$$

We rewrite $F_{\Gamma^{\prime} \Gamma}^{+}$in a form using the scattering matrix:

$$
\begin{aligned}
F_{\Gamma^{\prime} \Gamma}^{+}= & \sum_{j^{\prime}} c_{j^{\prime}}^{\Gamma^{\prime} \Gamma} \phi_{j^{\prime}}(r) \\
& +\sum_{l^{\prime} \lambda^{\prime}} \frac{i}{2}\left[h_{l^{\prime}}^{-}\left(k_{\Gamma^{\prime}} r\right) \delta_{l^{\prime} l} \delta_{\lambda^{\prime} \lambda} \delta_{\Gamma^{\prime} \Gamma}-S_{l^{\prime} \lambda^{\prime} ; \lambda^{\prime}}^{\Gamma^{\prime} \Gamma} h_{l^{\prime}}^{+}\left(k_{\Gamma^{\prime}} r\right)\right] \\
& \times Y_{l^{\prime} \lambda^{\prime}}(\hat{r}) / r
\end{aligned}
$$

with $\quad h_{l^{\prime}}^{-}\left(k_{\Gamma^{\prime}} r\right)=\left[h_{l^{\prime}}^{+}\left(k_{\Gamma^{\prime}} r\right)\right]^{*} \quad$ and $\quad S_{l^{\prime} \lambda^{\prime} ; l \lambda}^{\Gamma^{\prime}}=\delta_{l^{\prime} l} \delta_{\lambda^{\prime} \lambda} \delta_{\Gamma^{\prime} \Gamma}-$ $2 i T_{l^{\prime} \lambda ; l \lambda}^{\Gamma^{\prime} \Gamma}$ or, in matrix notations $\hat{S}=\hat{I}-2 i \hat{T}$, where $\hat{I}$ is the identity matrix and $\hat{S}$ is the scattering matrix. In terms of scattering matrix elements, the wave function $\Psi_{\Gamma l \lambda}$ can be written as

$$
\begin{aligned}
\Psi_{\Gamma l \lambda}= & \sum_{j} b_{j}^{\Gamma} \Theta_{j}+\hat{\mathcal{A}} \sum_{\Gamma^{\prime} j^{\prime}} \chi_{\Gamma^{\prime}} c_{j^{\prime}}^{\Gamma^{\prime} \Gamma} \phi_{j^{\prime}}(r)+\hat{\mathcal{A}} \sum_{\Gamma^{\prime} l^{\prime} \lambda^{\prime}} \omega_{\Gamma^{\prime}} Y_{l^{\prime} \lambda^{\prime}}(\hat{r}) \\
& \times \frac{i}{2 r}\left[h_{l^{\prime}}^{-}\left(k_{\Gamma^{\prime}} r\right) \delta_{l^{\prime} l} \delta_{\lambda^{\prime} \lambda} \delta_{\Gamma^{\prime} \Gamma}-S_{l^{\prime} \lambda^{\prime} ; l \lambda}^{\Gamma^{\prime} \Gamma} h_{l^{\prime}}^{+}\left(k_{\Gamma^{\prime}} r\right)\right] . \quad(10)
\end{aligned}
$$

The precision of TDM in Eq. (4) computed numerically using the complex-Kohn method is crucial to obtain an accurate final REA cross section. Because the calculation should describe accurately the $\mathrm{CN}+e^{-}$system, it is important to use a basis containing diffuse orbitals and we chose the large aug-cc-pVQZ basis with spd and $f$ orbitals. Note that the use of a smaller augmented basis, such as aug-cc-pVTZ without $f$ orbital, produces no appreciable change in the TDM. Using the latter basis, we describe the $\mathrm{CN}$ target through a complete active space (CAS) calculation in a reference space including ten natural orbitals of CN $(1 \sigma)(2 \sigma)(3 \sigma)(4 \sigma)\left(1 \pi_{x}\right)\left(1 \pi_{y}\right)(5 \sigma)\left(2 \pi_{x}\right)\left(2 \pi_{y}\right)(6 \sigma)$ and freezing two core orbitals. Note that the ground state $\Psi_{f}$ of $\mathrm{CN}^{-}$in Eq. (4) is calculated with a CAS in the same reference space as the $\mathrm{CN}$ target. Therefore, the affinity obtained from this calculation is usually smaller than the true affinity computed from more extensive calculations. One can drastically improve the affinity using optimized orbitals averaged over the $\mathrm{CN}-\mathrm{CN}^{-}$ground states in order to represent accurately the target and the negative ion. Using the latter orbitals in the calculations, we obtain a value as large as $3.1 \mathrm{eV}$ for zero-point affinity with no excitation outside the reference space, compared to $3.91 \mathrm{eV}$ with all single excitations allowed for $\mathrm{CN}^{-}$. We also obtained a vibrational frequency of $1962 \mathrm{~cm}^{-1}$ for $\mathrm{CN}$, and more importantly, an accurate value of $1.51 \mathrm{D}$ of the permanent dipole moment at the equilibrium position of $\mathrm{CN}$, whereas extensive calculations by Ajitha gave 1.49D [27] and the experimental value is 1.47D [28]. Moreover, the stability of the TDM (10-20\%) with respect to variation of different parameters in the calculation provides insight about the convergence and uncertainty of obtained results. For instance, the TDM have shown to be stable with respect to (i) the size of the reference space, (ii) freezing inner-shell orbitals, (iii) the size of the augmented basis, and (iv) the use of natural orbitals of $\mathrm{CN}$ (resulting in lower affinity) instead of averaged orbitals.

Besides computing the value of the TDMs in the MF in Eq. (4), it is instructive to look at the variation of the eigenphase sum of the scattering matrix as a function of the incident electron energy, shown in Fig. 2. The eigenphases, in both $\Sigma$ and $\Pi$ symmetries, are smoothly varying with increasing energy. The behavior of the eigenphases agrees well with the results of Ref. [16] obtained using the UK $R$-matrix code. It is clear from the figure that there is no shape resonances at low energy. No resonance means that one should expect a relatively small REA cross section, as it was already suggested by Harrison and Tennyson [16].

The TDMs have been calculated for eight values of $R$ in the interval $R=2.1-2.40 a_{0}$ with the grid step of $0.05 a_{0}$ and 


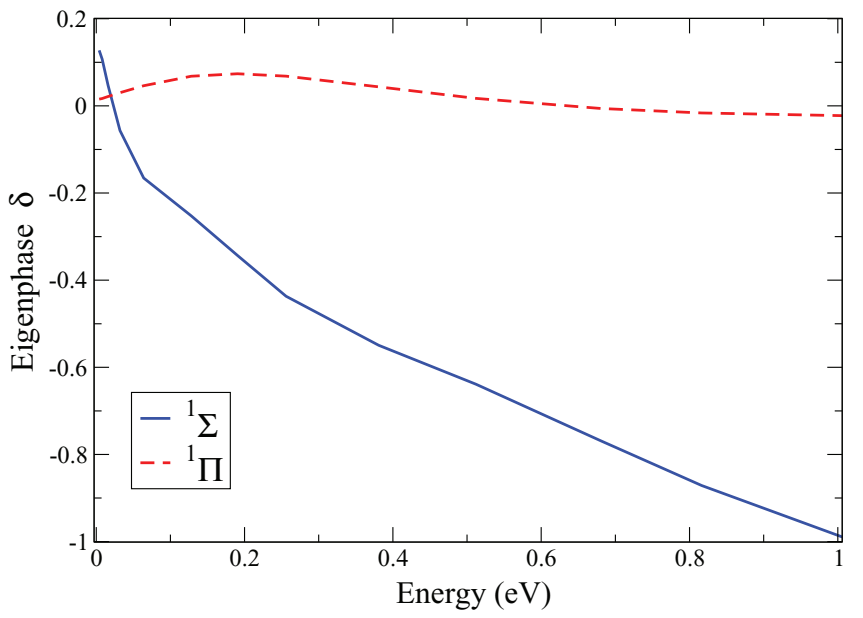

FIG. 2. (Color online) Eigenphase sum as a function of the incoming electron energy $E_{e l}$ (in $\mathrm{eV}$ ) for singlet ${ }^{1} \Sigma$ (solid line) and ${ }^{1} \Pi$ (dashed line) symmetries.

for eleven values of the electron energy, in the interval from $1 \mathrm{meV}$ to $1 \mathrm{eV}$ with a logarithmic step in energy. Figure 3 shows the dependence of dipole moments as a function of energy for an internuclear distance near the $\mathrm{CN}$ equilibrium. As expected, the $s$ wave produces the dominating transition dipole moment at low electron energy due to their penetrating character, whereas the contribution from higher partial waves

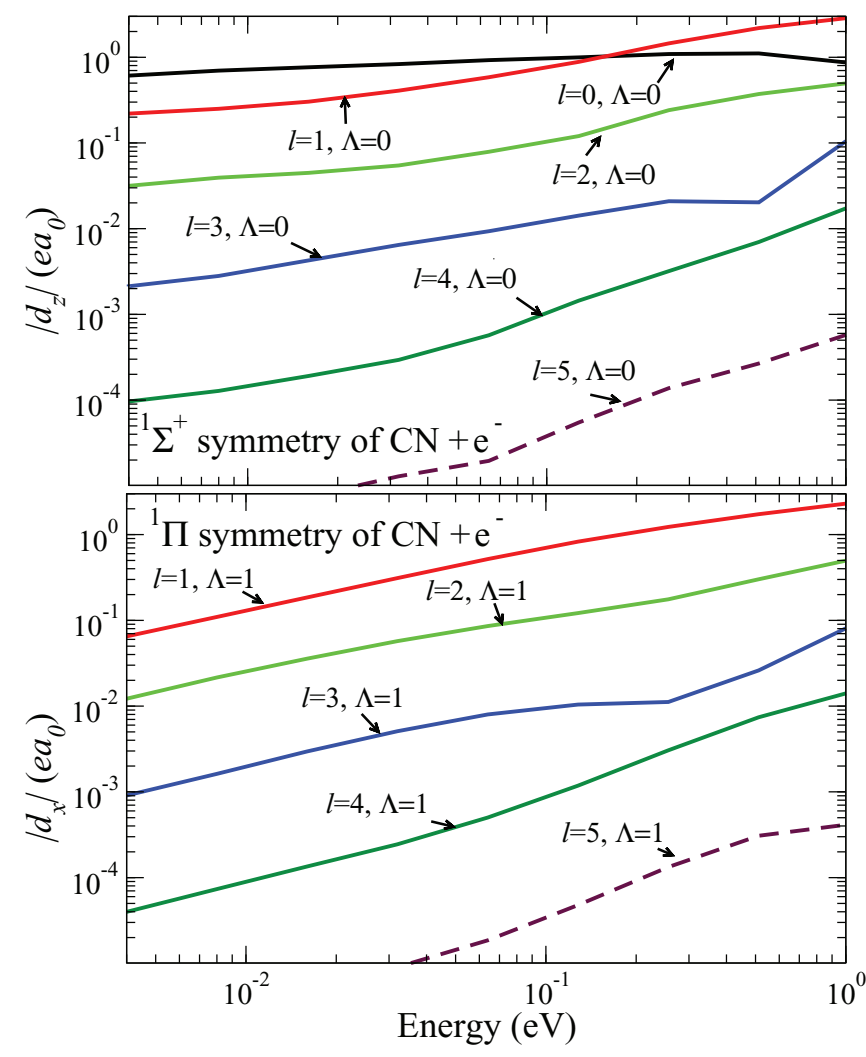

FIG. 3. (Color online) The energy dependence of the TDMs at $R=2.25 a_{0}$ for the Cartesian components $d_{z}$ (along the molecular axis) and $d_{x}$ (perpendicular to the axis). The figure shows the two components, $d_{z}=d_{0}$ and $d_{x}=\left(d_{-1}-d_{1}\right) / \sqrt{2}$. Because the initial $\mathrm{CN}$ state is a $\Sigma$ state, the projections $\Lambda$ and $\lambda$ are the same.

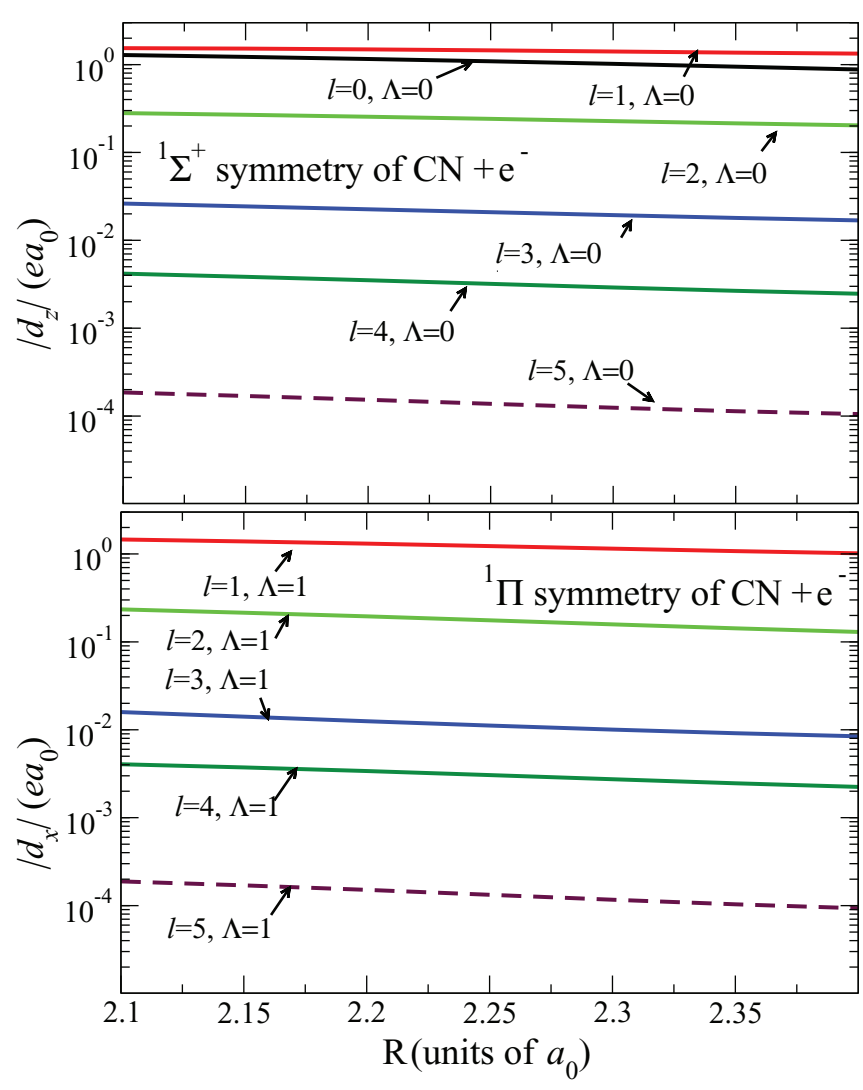

FIG. 4. (Color online) The figure shows the $d_{x}$ and $d_{z}$ components of the TDMs as a function of the internuclear distance $R$ for $\epsilon=$ $0.25 \mathrm{eV}$.

decreases considerably with the angular momentum $l$ and become negligible for $l \geqslant 2$. However, note that at larger electron energy, $p$ waves form the dominating transition-dipole moments. Finally, Fig. 4 depicts the weak dependence of the transition-dipole moments with respect to variation of the internuclear distance.

\section{RESULTS OF NUMERICAL CALCULATIONS}

The cross sections for REA from the initial rovibrational state $v_{i}, j$ of $\mathrm{CN}$ into the final state $v_{f}, J_{f}$ of $\mathrm{CN}^{-}$are calculated using Eq. (1). For astrophysical applications, the interest is in the sum $\sigma_{j}=\sum_{f} \sigma_{f i}$ over all possible final rovibrational states $f$. The cross sections with a change of the vibrational quantum number $v_{f} \neq v_{i}$ are much smaller than the cross sections with $v_{f}=v_{i}$ because the potentials of $\mathrm{CN}$ and $\mathrm{CN}^{-}$ are almost identical (see Fig. 1). At low temperatures, $T<$ $1000 \mathrm{~K}$ only $v_{i}=0$ is populated in the ISM. Figure 5 shows the obtained REA cross section as a function of the incident electron energy. At energies below $30 \mathrm{meV}$, the cross section decreases approximately as $1 / \sqrt{E_{e l}}$ because at low energies the dominant transition dipole moment is due to the $s$ wave in the incident electronic flux. This moment increases weakly with energy (see Fig. 3). With energy-independent transitiondipole moments, the cross section would decrease as $1 / E_{e l}$. At energies above $0.1 \mathrm{eV}$, the $p$ wave becomes the dominant one with the transition-dipole moment growing with energy faster 


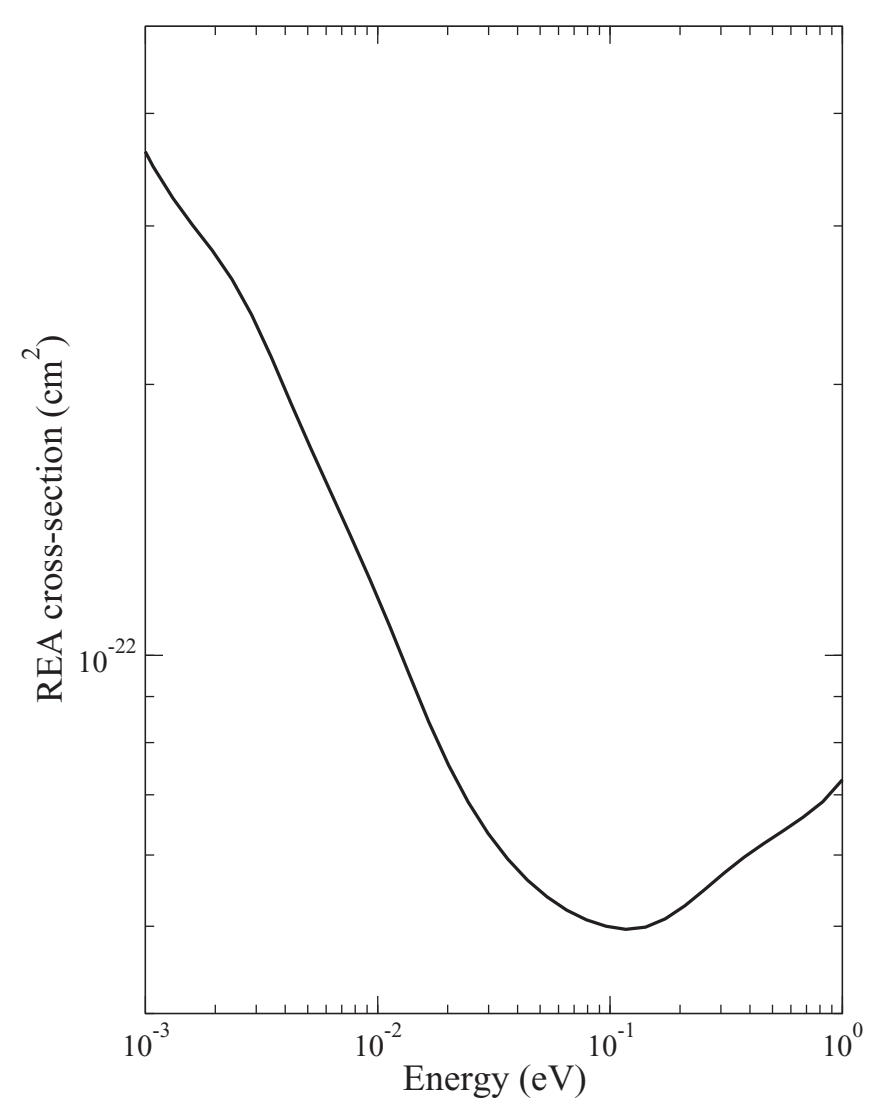

FIG. 5. The REA cross section $\sigma_{i}$ with the initial vibrational state of $\mathrm{CN} v_{i}=0$. A sum over all possible final rovibrational states of $\mathrm{CN}^{-}$ is performed. The contribution to the cross sections due to $v_{f}>0$ is negligible, less than $1 \%$.

than $\sqrt{E_{e l}}$. It leads to change in the cross section behavior: Above $0.1 \mathrm{eV}$ the cross section starts to grow with energy.

Recently, the photodetachment (PH) cross section of $\mathrm{CN}^{-}$was experimentally measured [29], which allows us to compare the results of the present study with the experiment. In [29], the photodetachment cross section for the process $\mathrm{CN}^{-}+\hbar \omega \rightarrow \mathrm{CN}+e^{-}$was obtained at the ultraviolet wavelength $266 \mathrm{~nm}(4.66 \mathrm{eV})$ and it was found that $\sigma=1.18 \times 10^{-17} \mathrm{~cm}^{2}$. Since photodetachment represents the inverse process of radiative attachment, one can get an estimation of the corresponding REA cross section using microscopic reversibility [30]:

$$
\sigma_{\mathrm{REA}}^{(\exp )}\left(E_{e l}\right)=\frac{g_{C N^{-}}}{g_{C N}} \frac{\hbar^{2} \omega^{2}}{2 m_{e} E_{e l} c^{2}} \sigma_{\mathrm{PH}}^{(\exp )}(\hbar \omega) .
$$

In the above expression, the statistical weights of $\mathrm{CN}^{-}\left(X^{1} \Sigma^{+}\right)$ and $\mathrm{CN}\left(X^{2} \Sigma^{+}\right)+e^{-}$are $g_{C N^{-}}=1$ and $g_{C N}=2$, respectively. Since the best measured experimental affinity of $\mathrm{CN}$ is $3.862 \pm 0.004 \mathrm{eV}[21]$, at a photon energy of $\hbar \omega=4.66 \mathrm{eV}$, the photoelectron energy is $E_{e l}=0.798 \mathrm{eV}$. Inserting these values in Eq. (11), we find the estimated value $\sigma_{\mathrm{REA}}^{(\exp )}\left(E_{e l}\right)=$ $1.57 \times 10^{-22} \mathrm{~cm}^{2}$, whereas our theoretical cross section $\sigma_{\text {REA }}^{(t h)}\left(E_{e l}\right)=6.8 \times 10^{-23} \mathrm{~cm}^{2}$ is about a factor of two lower. The good agreement between our theoretical values and the estimation obtained through microscopic reversibility confirms the overall precision of the present theoretical approach.

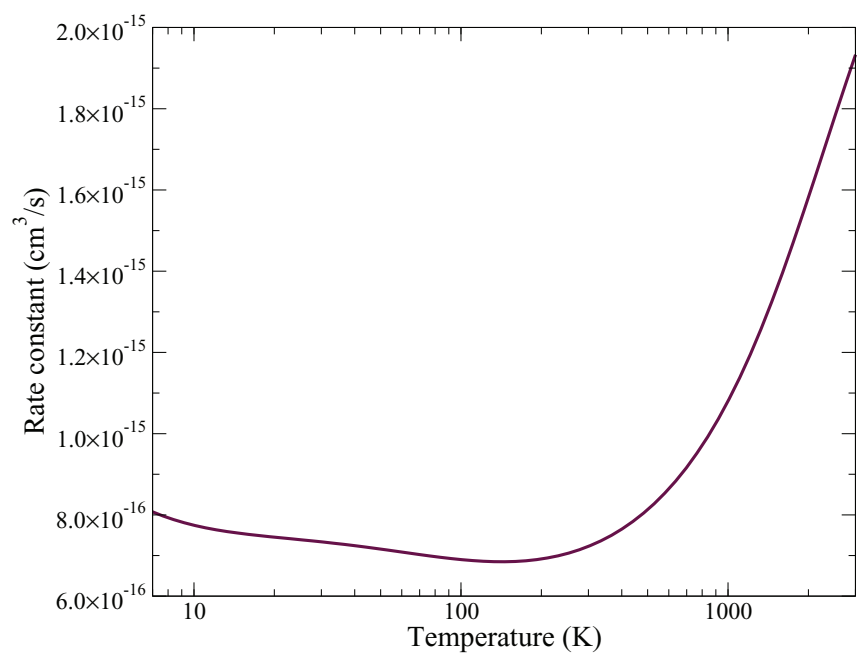

FIG. 6. (Color online) Thermally averaged rate coefficient $k(T)$.

Using the cross section, we calculate the thermally averaged rate coefficients $k(T)$ using the standard formula

$$
k(T)=\frac{8 \pi}{(2 k T)^{3 / 2}} \int_{0}^{\infty} \sigma(E) e^{-E / k T} E d E,
$$

which is shown in Fig. 6. The obtained rate coefficient is of the same order of magnitude as the one of the PST [4] for process (ii) discussed in Sec. II. However, the agreement is probably accidental, because PST assumes that the probability to capture the electron into an excited vibrational level of the negative ion is unity (the unitarity limit, see Eq. (12) or Ref. [4]). Our estimation showed that the probability for the electron capture is smaller by several orders of magnitude, thus leading to a much smaller REA rate coefficient for process (ii) than predicted by the PST.

\section{CONCLUSION}

We have developed a theory of radiative electron attachment to diatomic neutral molecules. The theory is based on first principles and accounts for rotational and vibrational degrees of freedom of the target molecule and the final negative molecular ion. The final formula for the REA cross section is relatively simple and involves vibrational integration of the TDM elements over the vibrational wave functions of the neutral molecule and the negative molecular ion. The TDM elements between the initial electronic continuum and final electronic bound states need to be calculated using scattering codes. In this study, we used the complex-Kohn variational method to determine the TDM matrix elements.

The theory has been applied to the formation of the $\mathrm{CN}^{-}$ ion by REA at collision energies below $1 \mathrm{eV}$. Cross sections and rate coefficients for $\mathrm{CN}^{-}$formation by REA have been evaluated. The obtained thermally averaged rate coefficient is relatively small and of the same order of magnitude as the one predicted by the phase-space theory, but the agreement in the order of magnitude should be considered as accidental.

At $300 \mathrm{~K}$, the present theory gives the REA rate coefficient $7 \times 10^{-16} \mathrm{~cm}^{3} / \mathrm{s}$. Modeling the formation of the $\mathrm{CN}^{-}$ion, detected [10] in the C-star envelope IRC +10216 , the authors of Ref. [10] used a larger value for the rate coefficient 
$2 \times 10^{-15} \mathrm{~cm}^{3} / \mathrm{s}$ at $300 \mathrm{~K}$ and obtained an agreement for the observed abundance and column density of $\mathrm{CN}^{-}$with the model. However, the spatial distribution of $\mathrm{CN}^{-}$is significantly different in the model and the fit derived from observation. The larger rate coefficient used in the model of Ref. [10] compared to what our study predicts, and the disagreement for the spatial distribution might indicate that the main mechanism for $\mathrm{CN}^{-}$formation in the circumstellar envelopes is not REA. There could be several other possibilities for the $\mathrm{CN}^{-}$formation near the central star as the observation demonstrated. Two such possibilities are the reaction $\mathrm{HCN}+\mathrm{H}^{-} \rightarrow \mathrm{CN}^{-}+\mathrm{H}_{2}$ or dissociative electron attachment to such molecules as $\mathrm{H}_{2} \mathrm{CN}$. The first reaction seems to be included into the model of Ref. [10], however, one should critically evaluate the rate constant for this reaction used in the model. The dissociative electron attachment as a source of $\mathrm{CN}^{-}$in circumstellar envelops has not been included, to the best of our knowledge, into the model but should be considered as a possible formation mechanism in the inner part of the circumstellar envelops.

The fully quantum REA theory can be extended to larger molecules. However, for larger molecules, one would need to determine potential energy surfaces and TDM in several dimensions corresponding to the number of vibrational degrees of freedom. Our preliminary calculation performed for two other negative molecular ions observed in the ISM, $\mathrm{C}_{3} \mathrm{~N}^{-}$, and $\mathrm{C}_{4} \mathrm{H}^{-}$, suggests that potential energy surfaces for the ground electronic states of the target molecule and the corresponding molecular ion are similar in shape near the equilibrium position of the target molecule. If the corresponding TDMs are weakly dependent on the geometry of the molecule near the equilibrium, similarly to the $\mathrm{CN}-\mathrm{CN}^{-}$case, a theoretical REA treatment for these systems could be greatly simplified. There could be, however, some additional effects in molecules larger than $\mathrm{CN}$. For example, the neutral $\mathrm{C}_{4} \mathrm{H}$ molecule has an excited electronic state ${ }^{2} \Pi$, which is very close in energy to the ground electronic state $X^{2} \Sigma^{+}[31]$ and should, therefore, be included into the theoretical treatment of REA in $\mathrm{C}_{4} \mathrm{H}$. Our preliminary calculations made for $\mathrm{C}_{3} \mathrm{~N}^{-}$and $\mathrm{C}_{4} \mathrm{H}^{-}$indicated that TDM matrix elements for these ions should not be significantly larger than in the case of $\mathrm{CN}^{-}$. More extensive calculations for $\mathrm{C}_{3} \mathrm{~N}^{-}$ and $\mathrm{C}_{4} \mathrm{H}^{-}$are in progress.

\section{ACKNOWLEDGMENTS}

This work is supported by the DOE Office of Basic Energy Science and the National Science Foundation, Grants No. PHY-11-60611 and No. PHY-10-68785. N.D. and S.F. are grateful for the time spent in the laboratoire Aimé Cotton and the University of Central Florida during part of this project. V.K. is also grateful to the Réseau thématique de recherches avancées "Triangle de la Physique", Contract No. QCCM-2008-007T for the partial support of the project at the initial stage.

\section{APPENDIX: DERIVATION OF THE FORMULA FOR REA CROSS SECTION}

We now derive the cross section formula in Eq. (1) and discuss the employed approximations. The starting point is to derive the initial electron-molecule wave function for an incoming low-energy electron scattering out of the rovibrating $\mathrm{CN}$ molecule. On the second step, we derive the probability of spontaneous emission from the initial state towards the ground state of $\mathrm{CN}^{-}$.

The rovibronic states of the target radical are expressed as follows:

$$
\phi_{v j m_{j}}^{\Gamma}=\sqrt{\frac{2 j+1}{8 \pi^{2}}}\left[D_{m_{j} \mu}^{j}(\Omega)\right]^{*} \chi_{v}^{j}(R) \omega_{\Gamma}\left(\mathbf{r}_{\mathbf{N}-\mathbf{1}}\right),
$$

where $j, m_{j}$, and $\mu$ are the angular momentum of $\mathrm{CN}$ and its projections in the LF and the MF correspondingly, $\chi_{v}^{j}(R)$ is the vibrational wave function of $\mathrm{CN}$ calculated using the molecular potential modified by the centrifugal term $\left[j(j+1)-\mu^{2}\right] /\left(2 \mu_{C N} R^{2}\right)$, where $\mu_{C N}$ is the reduced mass of $\mathrm{CN}$ and $R$ its internuclear distance. The function $\omega_{\Gamma}\left(\mathbf{r}_{\mathrm{N}-1}\right)$ specifies the $(N-1)$-electrons wave function of $\mathrm{CN}$ [see Eq. (6)] and $D_{m_{j} \mu}^{j}(\Omega)$ is the Wigner function depending on three Euler angles, which are called collectively by symbol $\Omega$.

For a given direction of propagation of an incoming electron and an initial state of $\mathrm{CN}$ defined by the quantum numbers $\left\{v, j, m_{j}, \Gamma\right\}$, the initial wave function of the system in the asymptotic region is given by

$$
\begin{aligned}
\Psi_{v j m_{j}}^{\Gamma} \rightarrow & e^{i \vec{k}_{v j} \cdot \vec{r}} \phi_{v j m_{j}}^{\Gamma}+\sum_{v^{\prime} j^{\prime} m_{j^{\prime}} \Gamma^{\prime}} \frac{e^{i k_{v^{\prime} j^{\prime}} r}}{r} A_{v j m_{j} \Gamma \rightarrow v^{\prime} j^{\prime} m_{j^{\prime}} \Gamma^{\prime}} \\
& \times\left(\vec{k}_{v j}, \vec{r}\right) \phi_{v^{\prime} j^{\prime} m_{j}}^{\Gamma^{\prime}}
\end{aligned}
$$

In the above expression, $\vec{k}_{v j}$ represents the asymptotic electronic wave vector in the energetically open channels labeled by the quantum numbers $v$ and $j$, and $A_{v j m_{j} \Gamma \rightarrow v^{\prime} j^{\prime} m_{j} \Gamma^{\prime}}\left(\vec{k}_{v j}, \vec{r}\right)$ denotes the differential amplitude for scattering from $\left\{v, j, m_{j}, \Gamma\right\}$ to $\left\{v^{\prime}, j^{\prime}, m_{j}^{\prime}, \Gamma^{\prime}\right\}$.

Because the decomposition of the plane wave in spherical waves is written as

$$
e^{i \vec{k}_{v j} \cdot \vec{r}}=4 \pi \sum_{l=0}^{\infty} \sum_{m_{l}=-l}^{l} i^{l} j_{l}\left(k_{v j} r\right) Y_{l m_{l}}\left(\hat{k}_{v j}\right) Y_{l m_{l}}(\hat{r}),
$$

where $j_{l}\left(k_{v j} r\right)$ is the regular Bessel function, the asymptotic form of the initial state in Eq. (A2) can be expressed in terms of $\hat{S}$-matrix elements

$$
\begin{aligned}
\Psi_{v j m_{j}}^{\Gamma} \rightarrow & \frac{2 \pi i}{r \sqrt{k_{v j}}} \sum_{l=0}^{\infty} \sum_{m_{l}=-l}^{l} Y_{l m_{l}}\left(\hat{k}_{v j}\right) i^{l} \\
& \times \sum_{l^{\prime} m_{l^{\prime}}} \sum_{v^{\prime} j^{\prime} m_{j}^{\prime} \Gamma^{\prime}} \frac{1}{\sqrt{k_{v^{\prime} j^{\prime}}}} \phi_{v^{\prime} j^{\prime} m_{j}^{\prime}} Y_{l^{\prime} m^{\prime}}(\hat{r}) \\
& \times\left[\delta_{v^{\prime} v} \delta_{l^{\prime} l} \delta_{m_{l^{\prime} m_{l}}} \delta_{j^{\prime} j} \delta_{m_{j^{\prime}} m_{j}} \delta_{\Gamma \Gamma^{\prime}} e^{-i\left(k_{v^{\prime} j^{\prime}} r-\frac{l^{\prime} \pi}{2}\right)}\right. \\
& \left.-S_{v^{\prime} j^{\prime} m_{j^{\prime}} \Gamma^{\prime} l_{l^{\prime}} ; v j m_{j} l m_{l}} e^{i\left(k_{v^{\prime} j^{\prime}} r-\frac{l^{\prime} \pi}{2}\right)}\right] .
\end{aligned}
$$

We use the rotational symmetry of the system and construct the following channel functions with total angular momentum 
$\vec{J}=\vec{l}+\vec{j}$ and projection $M=m_{l}+m_{j}$ in the $\mathrm{LF}$

$$
\begin{aligned}
\Phi_{v j l \Gamma}^{J M}= & \sqrt{\frac{2 j+1}{8 \pi^{2}}} \chi_{v}^{j}(R) \omega_{\Gamma}\left(\mathbf{r}_{\mathrm{N}-1}\right) \\
& \times \sum_{m_{l}=-l}^{l} \sum_{m_{j}=-j}^{j} C_{l m_{l} j m_{j}}^{J M}\left[D_{m_{j} \mu}^{j}(\Omega)\right]^{*} Y_{l m_{l}}(\hat{r}) .
\end{aligned}
$$

In terms of these channel functions, the function in Eq. (A4) takes the form

$$
\begin{aligned}
\Psi_{v j m_{j}}^{\Gamma} \rightarrow & \frac{2 \pi i}{r \sqrt{k_{v j}}} \sum_{J M} \sum_{l m_{l}} Y_{l m_{l}}\left(\hat{k}_{v j}\right) i^{l} \sum_{v^{\prime} j^{\prime} l^{\prime}} \frac{C_{l m_{l} j m_{j}}^{J M}}{\sqrt{k_{v^{\prime} j^{\prime}}}} \Phi_{v^{\prime} j^{\prime} l^{\prime} \Gamma^{\prime}}^{J M} \\
& \times\left[\delta_{v^{\prime} v} \delta_{l^{\prime} l} \delta_{j^{\prime} j} \delta_{\Gamma \Gamma^{\prime}} e^{-i\left(k_{v^{\prime} j^{\prime}} r-\frac{l^{\prime} \pi}{2}\right)}\right. \\
& \left.-S_{v^{\prime} j^{\prime} l^{\prime} \Gamma^{\prime} ; v j l \Gamma}^{J i\left(k_{v^{\prime} j^{\prime}} r-\frac{l^{\prime} \pi}{2}\right)}\right]
\end{aligned}
$$

It is important to note that in the above equation, the initial state of the target is still specified by the quantum numbers $\left\{v, j, m_{j}, \Gamma\right\}$. In order to express the above function in terms of MF parameters, we transform the channel functions $\Phi_{v j l \Gamma}^{J M}$ as follows. First we rewrite the spherical harmonic using bodyframe coordinates

$$
Y_{l m_{l}}(\theta, \phi)=\sum_{\lambda=-l}^{l} Y_{l \lambda}\left(\theta^{\prime}, \phi^{\prime}\right)\left[D_{m_{l} \lambda}^{l}(\Omega)\right]^{*},
$$

where $\lambda$ is the projection of the electron angular momentum on the molecular axis, $(\theta, \phi)$ and $\left(\theta^{\prime}, \phi^{\prime}\right)$ are spherical angles defined with respect to a fixed laboratory axis and the molecular axis, respectively. Then we make use of the fact that

$$
D_{m_{j} \mu}^{j}(\Omega) D_{m_{l} \lambda}^{l}(\Omega)=\sum_{J^{\prime}=|j-l|}^{j+l} D_{M \Lambda}^{J^{\prime}}(\Omega) C_{j m_{j} l m_{l}}^{J^{\prime} M} C_{j \mu l \lambda}^{J \Lambda},
$$

with $\Lambda=\mu+\lambda$, to rewrite the following term in Eq. (A5), at fixed $J$ and $M$, as

$$
\begin{aligned}
& \sum_{m_{j} m_{l}} C_{l m_{l} j m_{j}}^{J M}\left[D_{m_{j} \mu}^{j}(\Omega)\right]^{*} Y_{l m_{l}}(\theta, \phi) \\
& \quad=\sum_{m_{j} m_{l}} \sum_{J^{\prime} \lambda}\left[D_{M \Lambda}^{J^{\prime}}(\Omega)\right]^{*} C_{j m_{j} l m_{l}}^{J M} C_{j m_{j} l m_{l}}^{J^{\prime} M} C_{j \mu l \lambda}^{J^{\prime} \Lambda} Y_{l \lambda}\left(\theta^{\prime}, \phi^{\prime}\right) .
\end{aligned}
$$

From the orthonormality relation

$$
\sum_{m_{j} m_{l}} C_{j m_{j}, l m_{l}}^{J^{\prime} M^{\prime}} C_{j m_{j}, l m_{l}}^{J M}=\delta_{J^{\prime} J} \delta_{M^{\prime} M},
$$

this equation simply becomes

$$
\begin{aligned}
& \sum_{m_{j} m_{l}} C_{l m_{l} j m_{j}}^{J M}\left[D_{m_{j} \mu}^{j}(\Omega)\right]^{*} Y_{l m_{l}}(\theta, \phi) \\
& \quad=\sum_{\lambda} C_{j \mu l \lambda}^{J \Lambda}\left[D_{M \Lambda}^{J}(\Omega)\right]^{*} Y_{l \lambda}\left(\theta^{\prime}, \phi^{\prime}\right) \\
& \quad=\sum_{\lambda} \sqrt{\frac{2 J+1}{2 j+1}}(-1)^{l+\lambda} C_{l-\lambda J \Lambda}^{j \mu}\left[D_{M \Lambda}^{J}(\Omega)\right]^{*} Y_{l \lambda}\left(\theta^{\prime}, \phi^{\prime}\right) .
\end{aligned}
$$

We now naturally introduce body-fixed channel functions denoted $X_{v l \lambda}^{J M}$

$$
X_{v l \lambda \Gamma}^{J M}=\sqrt{\frac{2 J+1}{8 \pi^{2}}} \chi_{v}^{j}(R) \omega_{\Gamma}\left(\mathbf{r}_{\mathbf{N}-\mathbf{1}}\right)\left[D_{M \Lambda}^{J}(\Omega)\right]^{*} Y_{l \lambda}\left(\theta^{\prime}, \phi^{\prime}\right) .
$$

The channel functions $X_{v l \lambda}^{J M}$ transform into $\Phi_{v j l}^{J M}$ in the following way

$$
\begin{aligned}
& X_{v l \lambda \Gamma}^{J M}=\sum_{j} \Phi_{v j l \Gamma}^{J M} U_{j \lambda}^{J M} \text { and } \\
& \Phi_{v j l \Gamma}^{J M}=\sum_{\lambda} X_{v l \lambda \Gamma}^{J M} U_{\lambda j}^{J M \dagger}
\end{aligned}
$$

where the elements of the unitarity matrix $\hat{U}$ are simply given by

$$
U_{j \lambda}^{J M}=(-1)^{l+\lambda} C_{l-\lambda J \Lambda}^{j \mu} .
$$

The above transformation is readily applied on the wave function of Eq. (A6), namely, changing from the $j$ representation to the $\lambda$ representation:

$$
\begin{aligned}
& \sum_{j^{\prime}} S_{v^{\prime} j^{\prime} l^{\prime} \Gamma^{\prime} ; v j l \Gamma}^{J} \Phi_{v^{\prime} j^{\prime} l^{\prime} \Gamma^{\prime}}^{J M} \\
& \quad=\sum_{j^{\prime}} \sum_{\lambda^{\prime}} \sum_{\lambda} U_{j^{\prime} \lambda^{\prime}}^{J M} S_{v^{\prime} l^{\prime} \lambda^{\prime} \Gamma^{\prime} ; v l \lambda \Gamma}^{J} U_{\lambda j}^{J M^{\dagger}} \Phi_{v^{\prime} j^{\prime} l^{\prime} \Gamma^{\prime}}^{J M} \\
& \quad=\sum_{\lambda^{\prime}} \sum_{\lambda} U_{j \lambda}^{J M} S_{v^{\prime} l^{\prime} \lambda^{\prime} ; v l \lambda}^{J} X_{v^{\prime} l^{\prime} \lambda^{\prime} \Gamma^{\prime}}^{J M} .
\end{aligned}
$$

The initial state in terms of MF channels takes the form:

$$
\begin{aligned}
\Psi_{v j m_{j}}^{\Gamma} \rightarrow & \frac{2 \pi i}{r \sqrt{k_{v j}}} \sum_{J M \lambda} \sum_{l m_{l}} Y_{l m_{l}}\left(\hat{k}_{v j}\right) i^{l} \\
& \times \sum_{v^{\prime} l^{\prime} \lambda^{\prime} \Gamma^{\prime}}(-1)^{l+\lambda} \frac{C_{l m_{l} j m_{j}}^{J M} C_{l-\lambda J \Lambda}^{j \mu}}{\sqrt{k_{v^{\prime} j}}} X_{v^{\prime} l^{\prime} \lambda^{\prime} \Gamma^{\prime}}^{J M} \\
& \times\left[\delta_{\lambda^{\prime} \lambda} \delta_{v^{\prime} v} \delta_{l^{\prime} l} \delta_{\Gamma \Gamma^{\prime}} e^{-i\left(k_{v^{\prime} j} r-\frac{l^{\prime} \pi}{2}\right)}\right. \\
& \left.-S_{v^{\prime} l^{\prime} \lambda^{\prime} \Gamma^{\prime} ; v l \lambda \Gamma}^{J} e^{i\left(k_{v^{\prime} j} r-\frac{l^{\prime} \pi}{2}\right)}\right] .
\end{aligned}
$$

Note that the transformation from Eq. (A6) to Eq. (A16) is exact only if the channels, which are mixed, are degenerate in energy. It is needed in order to have $k_{v^{\prime} j^{\prime}} \approx k_{v^{\prime} j}$ for any $j^{\prime}$. The reason is that the asymptotic energy of the system at $r \rightarrow \infty$ is determined by the quantum number $j$, which is well defined for channels $\Phi_{v j l \Gamma}^{J M}$ but not for $X_{v l \lambda \Gamma}^{J M}$. The wave vectors are approximately equal to each other for large electron energies $E_{e l} \gg B_{v}$, where $B_{v}$ is the rotational constant of the target or if they are evaluated at a finite distance $r$ such that $E_{e l}-V(r) \gg B_{v}$, where $V(r)$ is the potential of interaction of the electron with the target at large values of $r$. For $\mathrm{CN}$, $V(r)$ behaves as $d_{e} / r^{2}$ with $d_{e} \sim 1$ and $B_{v} \sim 10^{-5}$ in atomic units. In the discussion below, we assume that the collision energy is high enough to satisfy the condition $E_{e l} \gg B_{v}$, which is the case for most of the astrophysical environments. For smaller energies, the present approach can be improved by using the multichannel quantum defect approach in which an intermediate, so-called smooth, scattering matrix is obtained at a distance $r$ where $E_{e l}-V(r) \gg B_{v}$ and then it is used to 
calculate the actual physically meaningful scattering matrix using the channel elimination procedure.

We finally apply the vibrational-frame transformation on Eq. (A16), changing from the $v$ representation to the $R$ representation

$$
\begin{aligned}
& \sum_{v^{\prime}} S_{v^{\prime} l^{\prime} \lambda^{\prime} ; v l \lambda}^{J} \chi_{v^{\prime}}^{j}(R) \\
& \quad=\sum_{v^{\prime}} \int \chi_{v^{\prime}}^{j}(R) \chi_{v^{\prime}}^{j}\left(R^{\prime}\right) S_{l^{\prime} \lambda^{\prime} ; l \lambda}^{\Gamma^{\prime} \Gamma}\left(R^{\prime}\right) \chi_{v}^{j}\left(R^{\prime}\right) d R^{\prime} \\
& \quad=S_{l^{\prime} \lambda^{\prime} ; l \lambda}^{\Gamma^{\prime} \Gamma}(R) \chi_{v}(R),
\end{aligned}
$$

where we have applied the closure relation $\sum_{v^{\prime}} \chi_{v^{\prime}}^{j}\left(R^{\prime}\right) \chi_{v^{\prime}}^{j}(R)=\delta\left(R^{\prime}-R\right)$ and introduce the smooth $R$-dependent scattering matrix $S_{l^{\prime} \lambda^{\prime} ; l \lambda}^{\Gamma^{\prime}}(R)$ calculated through the complex-Kohn method. The initial state now becomes

$$
\begin{aligned}
\Psi_{v j m_{j}}^{\Gamma} \rightarrow & \frac{2 \pi i}{r \sqrt{k_{v j}}} \sum_{J M \lambda} \sum_{l m_{l}} Y_{l m_{l}}\left(\hat{k}_{v j}\right) i^{l}(-1)^{l+\lambda} \frac{C_{l m_{l} j m_{j}}^{J M} C_{l-\lambda J \Lambda}^{j \mu}}{\sqrt{k_{v j}}} \\
& \times \sum_{l^{\prime} \lambda^{\prime} \Gamma^{\prime}} X_{v l^{\prime} \lambda^{\prime} \Gamma^{\prime}}^{J M}\left[\delta_{l^{\prime} l} \delta_{\lambda^{\prime} \lambda} \delta_{\Gamma^{\prime} \Gamma} e^{-i\left(k_{v j} r-\frac{l^{\prime} \pi}{2}\right)}\right. \\
& \left.-S_{l^{\prime} \lambda^{\prime} ; l \lambda}^{\Gamma^{\prime} \Gamma}(R) e^{i\left(k_{v j} r-\frac{l^{\prime} \pi}{2}\right)}\right] .
\end{aligned}
$$

The asymptotic behavior of Eq. (A18) can now be used to express the entire electronic function with the help of Eq. (10) and the Born-Oppenheimer approximation. We introduce the notation $|J M \Lambda\rangle=\sqrt{\frac{2 J+1}{8 \pi^{2}}}\left[D_{M \Lambda}^{J}(\Omega)\right]^{*}$ for the rotational state of the $\mathrm{CN}+e^{-}$system and simplify the expression by considering the $\tilde{z}$ axis of the $\mathrm{LF}$ as the direction of the incoming wave vector $\vec{k}_{v j}$. Using this convention, we have $m_{l}=0$ and since $Y_{l 0}(\tilde{z})=\sqrt{(2 l+1) / 4 \pi}$, the initial state becomes

$$
\begin{aligned}
\Psi_{v j m_{j}}^{\Gamma}= & \frac{1}{\sqrt{k_{v j}}} \sum_{J l \lambda} \sqrt{4 \pi(2 l+1)} i^{l}(-1)^{l+\lambda} C_{l 0 j m_{j}}^{J M} C_{l-\lambda J \Lambda}^{j \mu} \mid J \\
& \times \chi_{v}^{j}(R) \Psi_{\Gamma l \lambda}(r, R) .
\end{aligned}
$$

The approximations, which were employed in the above development, were needed in order for the MF electronic function to appear explicitly in the scattering state, which allows the further transformation from the MF transition-dipole moments to the LF transition-dipole moments. Therefore, the rovibronic interactions are taken into account in our treatment, but we neglect the difference in energy between different rotational states of $\mathrm{CN}$, such that rotational excitation of the target neutral molecule is not included in our approach, ultimately resulting in the absence of threshold structures in our REA cross section.

The initial continuum state can spontaneously decay to final states of $\mathrm{CN}^{-}$, which has the form

$$
\Psi_{v_{f} \Lambda_{f}}^{J_{f} M_{f}}=\sqrt{\frac{2 J_{f}+1}{8 \pi^{2}}}\left[D_{M_{f} \Lambda_{f}}^{J_{f}}(\Omega)\right]^{*} \chi_{v_{f}}^{J_{f}}(R) \Psi_{f}\left(\mathbf{r}_{\mathbf{N}}\right),
$$

where $J_{f}, M_{f}$, and $\Lambda_{f}$ are the angular momentum of $\mathrm{CN}^{-}$and its projections in the LF and in MF correspondingly, $\chi_{v_{f}}^{J_{f}}(R)$ is the vibrational wave function of $\mathrm{CN}^{-}$calculated using the molecular potential modified by the centrifugal term $\left[J_{f}\left(J_{f}+\right.\right.$ 1) $\left.-\Lambda_{f}^{2}\right] /\left(2 \mu_{C N} R^{2}\right)$. The state $\Psi_{f}$ specifies the electronic state of $\mathrm{CN}^{-}$. In the present study, it is always $X^{1} \Sigma^{+}$, i.e.,
$\Lambda_{f}=0$. In order to simplify the following development, we use the notation $|i\rangle$ for the initial state $\Psi_{v j m_{j}}^{\Gamma}$ and $|f\rangle$ for the final state $\Psi_{v_{f} \Lambda_{f}}^{J_{f} M_{f}}$. We now calculate the transition-dipole moments in the LF

$$
|\langle f|\vec{d}| i\rangle|^{2}=\sum_{p}\left|\left\langle f\left|d_{p}\right| i\right\rangle\right|^{2},
$$

where the sum is taken over the three spherical components $p=-1,0,+1$ of the dipole moment vector $\vec{d}$. The relationship between components of $\vec{d}$ in LF ( $d_{p}$ components) and MF ( $d_{\pi}$ components) is

$$
d_{p}=\sum_{\pi} d_{\pi}\left[D_{p \pi}^{1}(\Omega)\right]^{*}
$$

Therefore,

$$
\begin{aligned}
& \left\langle f\left|d_{p}\right| i\right\rangle \\
& =\frac{1}{\sqrt{k_{v j}}} \sum_{J l \lambda} C_{l 0 j m_{j}}^{J M} C_{l-\lambda J \Lambda}^{j \mu} \sqrt{4 \pi(2 l+1)} i^{l} \\
& \quad \times \sum_{\pi}(-1)^{l+\lambda} \int \chi_{v_{f}}^{J_{f}}(R)\left\langle\Psi_{f}\right|\left\langle J_{f} M_{f} \Lambda_{f}\right| \hat{d}_{\pi}\left[D_{p \pi}^{1}(\Omega)\right]^{*} \\
& \quad \times|J M \Lambda\rangle_{\Omega}\left|\Psi_{\Gamma l \lambda}\right\rangle_{r} \chi_{v}^{j}(R) d R .
\end{aligned}
$$

In the above formula, the subscripts $r$ and $\Omega$ symbolize the variables of integration, but the integral over $R$ is written explicitly. The integral over $\Omega$ in Eq. (A23) is evaluated using Eq. (5) of Sec 4.11 from Ref. [19]

$$
\left\langle J_{f} M_{f} \Lambda_{f}\left|\left[D_{p \pi}^{1}(\Omega)\right]^{*}\right| J M \Lambda\right\rangle=\sqrt{\frac{2 J+1}{2 J_{f}+1}} C_{J M 1 p}^{J_{f} M_{f}} C_{J \Lambda 1 \pi}^{J_{f} \Lambda_{f}} .
$$

To simplify notations and evaluation of integrals, we rewrite Eq. (A23) in a different form

$$
\left\langle f\left|d_{p}\right| i\right\rangle=\frac{1}{\sqrt{k_{v j}}} \sum_{J l} C_{l 0 j m_{j}}^{J M} \sqrt{\frac{2 J+1}{2 J_{f}+1}} C_{J M 1 p}^{J_{f} M_{f}} A_{J l \mu}^{\left(v \rightarrow v_{f}\right)},
$$

where we introduced the factors

$$
\begin{aligned}
A_{J l \mu}^{\left(v \rightarrow v_{f}\right)}= & \sum_{\lambda \pi} C_{J \Lambda 1 \pi}^{J_{f} \Lambda_{f}} C_{l-\lambda J \Lambda}^{j \mu} \sqrt{4 \pi(2 l+1)} i^{l}(-1)^{l+\lambda} \\
& \times \int \chi_{v_{f}}^{J_{f}}(R)\left\langle\Psi_{f}\left|d_{\pi}\right| \Psi_{\Gamma l \lambda}\right\rangle_{r} \chi_{v}^{j}(R) d R \\
= & \sum_{\lambda \pi} C_{J \Lambda 1 \pi}^{J_{f} \Lambda_{f}} C_{l-\lambda J \Lambda}^{j \mu} \sqrt{4 \pi(2 l+1)} i^{l}(-1)^{l+\lambda} d_{\pi, \Gamma l \lambda}^{\left(v \rightarrow v_{f}\right)},
\end{aligned}
$$

such that $A_{J l \mu}^{\left(v \rightarrow v_{f}\right)}$ does not depend on projections in LF. The matrix element of the transition-dipole moment is

$$
d_{\pi, \Gamma l \lambda}^{\left(v \rightarrow v_{f}\right)}=\int \chi_{v_{f}}^{J_{f}}(R)\left\langle\Psi_{f}\left|d_{\pi}\right| \Psi_{\Gamma l \lambda}\right\rangle_{r} \chi_{v}^{j}(R) d R .
$$

As a next step, we evaluate the sum over $p$ in Eq. (A21) and take another sum over possible final projections $M_{f}$. Because of the dipole moment components of Eq. (A25) enter Eq. (A21) as absolute values squared, the sum over $l$ and $J$ are 
doubled,

$$
\begin{aligned}
\sum_{M_{f}}|\langle f|\vec{d}| i\rangle|^{2}= & \frac{1}{k_{v j}} \sum_{J J^{\prime} M_{f} p} C_{J M 1 p}^{J_{f} M_{f}} C_{J^{\prime} M 1 p}^{J_{f} M_{f}} \sum_{l l^{\prime}} C_{l 0 j m_{j}}^{J M} C_{l^{\prime} 0 m_{j}}^{J^{\prime} M} \\
& \times \sqrt{\frac{(2 J+1)\left(2 J^{\prime}+1\right)}{\left(2 J_{f}+1\right)^{2}}}\left[A_{J^{\prime} l^{\prime} \mu}^{\left(v \rightarrow v_{f}\right)}\right]^{*} A_{J l \mu}^{\left(v \rightarrow v_{f}\right)} .
\end{aligned}
$$

The orthogonality of Clebsch-Gordan coefficients (Eq. (5) of Sec. 8.7.2 from Ref. [19]) allows us to evaluate sums over $p, M_{f}$, and $J^{\prime}$ in the above equation,

$$
\sum_{M_{f}}|\langle f|\vec{d}| i\rangle|^{2}=\frac{1}{k_{v j}} \sum_{J} \sum_{l l^{\prime}} C_{l 0 j m_{j}}^{J M} C_{l^{\prime} 0 j m_{j}}^{J M}\left[A_{J l^{\prime} \mu}^{\left(v \rightarrow v_{f}\right)}\right]^{*} A_{J l \mu}^{\left(v \rightarrow v_{f}\right)}
$$

On the next step, we average over initial orientation of the molecule in LF, i.e., over $m_{j}$, which is the same as $M$ :

$$
\begin{aligned}
& \frac{1}{2 j+1} \sum_{M M_{f}}|\langle f|\vec{d}| i\rangle|^{2} \\
& =\frac{1}{k_{v j}} \sum_{J} \frac{1}{2 j+1} \sum_{M l l^{\prime}} C_{l 0 j m_{j}}^{J M} C_{l^{\prime} 0 j m_{j}}^{J M}\left[A_{J l^{\prime} \mu}^{\left(v \rightarrow v_{f}\right)}\right]^{*} A_{J l \mu}^{\left(v \rightarrow v_{f}\right)} .
\end{aligned}
$$

Using again Eq. (5) of Sec. 8.7.2 from Ref. [19], we can evaluate the sum over $M$ and eliminate the sun over $l^{\prime}$

$$
\begin{aligned}
& \frac{1}{2 j+1} \sum_{M M_{f}}|\langle f|\vec{d}| i\rangle|^{2} \\
& =\frac{1}{k_{v j}} \sum_{J l} \frac{2 J+1}{(2 j+1)(2 l+1)}\left|A_{J l \mu}^{\left(v \rightarrow v_{f}\right)}\right|^{2} \\
& =\frac{4 \pi}{k_{v j}} \sum_{J l} \frac{2 J+1}{2 j+1}\left|\sum_{\lambda \pi}(-1)^{l+\lambda} C_{J \Lambda 1 \pi}^{J_{f} \Lambda_{f}} C_{l-\lambda J \Lambda}^{j \mu} d_{\pi, \Gamma l \lambda}^{\left(v \rightarrow v_{f}\right)}\right|^{2} .
\end{aligned}
$$

The probability $P_{i \rightarrow f}$ of spontaneous emission is given by

$$
P_{i \rightarrow f}=\frac{4}{3} \frac{\omega^{3}}{\hbar c^{3}}|\langle f|\vec{d}| i\rangle|^{2}
$$

and the cross section is obtained dividing the probability $P_{i \rightarrow f}$ with the density of current in the incident wave, which is equal to the velocity $v=\hbar k_{v j} / m_{e}$ in the incident wave of Eq. (A2). Therefore, the cross section for the radiative attachment to a given angular momentum $J_{f}$ is

$$
\begin{aligned}
\sigma_{f i}= & \frac{16}{3} \frac{\pi \omega^{3} m_{e}}{k_{v j}^{2} \hbar^{2} c^{3}} \sum_{J l} \frac{2 J+1}{2 j+1} \\
& \times\left|\sum_{\lambda \pi}(-1)^{l+\lambda} C_{J \Lambda 1 \pi}^{J_{f} \Lambda_{f}} C_{l-\lambda J \Lambda}^{j \mu} d_{\pi, \Gamma l \lambda}^{\left(v \rightarrow v_{f}\right)}\right|^{2},
\end{aligned}
$$

or in an equivalent form

$$
\sigma_{f i}=\frac{16}{3} \frac{\pi \omega^{3} m_{e}}{k_{v j}^{2} \hbar^{2} c^{3}} \sum_{J l}\left|\sum_{\lambda \pi} C_{J \Lambda 1 \pi}^{J_{f} \Lambda_{f}} C_{l \lambda j \mu}^{J \Lambda} d_{\pi, \Gamma l \lambda}^{\left(v \rightarrow v_{f}\right)}\right|^{2} .
$$

The coefficient in front of the formula above is different from other studies of radiative association and attachment (see, for example, Ref. [32]). The reason is that we use a particular normalization of the incident wave given by Eq. (8), which is not the same as in other studies on radiative association or attachment.

The spin-orbit interaction was neglected in the above treatment. Treating spin degrees of freedom statistically, we have to include the degeneracy factors $g_{f}$ and $g_{i}$, which are the number of final and initial states, respectively.

$\sigma_{f i}=\frac{16}{3} \frac{g_{f}}{g_{i}} \frac{\pi \omega^{3} m_{e}}{k_{v j}^{2} \hbar^{2} c^{3}} \sum_{J l}\left|\sum_{\lambda \pi} C_{J \Lambda 1 \pi}^{J_{f} \Lambda_{f}} C_{l \lambda j \mu}^{J \Lambda} d_{\pi, \Gamma l \lambda}^{\left(v \rightarrow v_{f}\right)}\right|^{2}$.

For REA of $\mathrm{CN}$, the number of initial spin states is four and there is only one final state, because the final $\mathrm{CN}^{-}$is a singlet state, thus $g_{f} / g_{i}=1 / 4$.

In order to calculate the REA cross section into all possible rotational states of $\mathrm{CN}^{-}$, one should take a sum over $J_{f}$. For $\Sigma^{+}$symmetry for initial and final states, it is possible to simplify the sum. For CN-CN ${ }^{-} \mu=\Lambda_{f}=0$ and, therefore, $\lambda=-\pi$, and $\Lambda=\lambda$ in Eq. (A35). The cross section (A35) summed over $J_{f}$ can then be written as

$$
\begin{aligned}
\sigma_{i}= & \sum_{J_{f}} \sigma_{f i} \\
= & \frac{4}{3} \frac{\pi \omega^{3} m_{e}}{k_{v j}^{2} \hbar^{2} c^{3}} \sum_{J_{f} J l} \sum_{\lambda \lambda^{\prime}} C_{J \lambda 1-\lambda}^{J_{f} 0} C_{J \lambda^{\prime} 1-\lambda^{\prime}}^{J_{f} 0} C_{l \lambda j 0}^{J \lambda} C_{l \lambda^{\prime} j 0}^{J \lambda^{\prime}} \\
& \times d_{-\lambda, \Gamma l \lambda}^{\left(v \rightarrow v_{f}\right)} d_{-\lambda^{\prime}, \Gamma l \lambda^{\prime}}^{\left(v \rightarrow \lambda_{f}\right) *}
\end{aligned}
$$

Because

$$
\sum_{J_{f}} C_{J \lambda 1-\lambda}^{J_{f} 0} C_{J \lambda^{\prime} 1-\lambda^{\prime}}^{J_{f} 0}=\delta_{\lambda \lambda^{\prime}}
$$

the cross section can be written as

$$
\sigma_{i}=\frac{4}{3} \frac{\pi \omega^{3} m_{e}}{k_{v j}^{2} \hbar^{2} c^{3}} \sum_{J l \lambda} C_{l \lambda j 0}^{J \lambda} C_{l \lambda j 0}^{J \lambda}\left|d_{-\lambda, \Gamma l \lambda}^{\left(v \rightarrow v_{f}\right)}\right|^{2} .
$$

Using the orthonormality of Clebsch-Gordan coefficients one more time

$$
\sum_{J} C_{l \lambda j 0}^{J \lambda} C_{l \lambda j 0}^{J \lambda}=1
$$

we obtain

$$
\begin{aligned}
\sigma_{i} & =\frac{4}{3} \frac{\pi \omega^{3} m_{e}}{k_{v j}^{2} \hbar^{2} c^{3}} \sum_{l \lambda}\left|d_{-\lambda, \Gamma l \lambda}^{\left(v \rightarrow v_{f}\right)}\right|^{2} \\
& =\frac{4}{3} \frac{\pi \omega^{3} m_{e}}{k_{v j}^{2} \hbar^{2} c^{3}} \sum_{l \pi}\left|d_{\pi, \Gamma l-\pi}^{\left(v \rightarrow v_{f}\right)}\right|^{2} .
\end{aligned}
$$

In our theoretical treatment of REA in $\mathrm{CN}$ we use the above formula. It is important to stress that when this formula is used for low energies such that some of the included rotational or vibrational channels of the $\mathrm{CN}+e^{-}$system are asymptotically closed, it is assumed that closed channels do not have resonances at the energy of interest. This condition is satisfied: the electronically bound states associated with an excited (closed) channel are about $3.8 \mathrm{eV}$ below the 
channel energy, i.e., they are situated deeply in the discrete spectrum. This statement can be formulated more rigorously in terms of quantum defect theory: When some channels are closed at a given energy, one should apply the closed-channel elimination procedure (see Eqs. (2.50) and (2.58) of Ref. [33]).
The channel elimination will significantly modify the dipole moment components in open channels only if the coupling between channels is strong or there is a resonance in a closed channel at a given energy. None of these conditions is satisfied for the case for REA in $\mathrm{CN}$ at collision energies below $1 \mathrm{eV}$.
[1] E. Herbst, Nature (London) 289, 656 (1981).

[2] M. C. McCarthy, C. A. Gottlieb, H. Gupta, and P. Thaddeus, Astrophys. J. Lett. 652, L141 (2006).

[3] J. Cernicharo, M. Guélin, M. Agúndez, K. Kawaguchi, M. McCarthy, and P. Thaddeus, Astron. Astrophys. 467, L37 (2007).

[4] E. Herbst and Y. Osamura, Astrophys. J. 679, 1670 (2008).

[5] N. Harada and E. Herbst, Astrophys. J. 685, 272 (2008).

[6] J. Cernicharo, M. Guélin, M. Agúndez, M. C. McCarthy, and P. Thaddeus, Astrophys. J. Lett. 688, L83 (2008).

[7] H. Gupta, S. Brünken, F. Tamassia, C. A. Gottlieb, M. C. McCarthy, and P. Thaddeus, Astrophys. J. Lett. 655, L57 (2007).

[8] K. Kawaguchi, R. Fujimori, S. Aimi, S. Takano, E. Y. Okabayashi, H. Gupta, S. Bruenken, C. A. Gottlieb, M. C. Mccarthy, and P. Thaddeus, Publ. Astron. Soc. Japan 59, L47 (2007).

[9] P. Thaddeus, C. A. Gottlieb, H. Gupta, S. Brünken, M. C. McCarthy, M. Agúndez, M. Guélin, and J. Cernicharo, Astrophys. J. 677, 1132 (2008).

[10] M. Agúndez, J. Cernicharo, M. Guélin, C. Kahane, E. Roueff, J. Kłos, F. J. Aoiz, F. Lique, N. Marcelino, J. R. Goicoechea et al., Astron. Astrophys. 517, L2 (2010).

[11] S. Petrie, Mon. Not. R. Astron. Soc. 281, 137 (1996).

[12] E. Herbst, Astron. Astrophys. 153, 151 (1985).

[13] R. Terzieva and E. Herbst, Int. J. Mass. Spectrom. 201, 135 (2000).

[14] S. Petrie and E. Herbst, Astrophys. J. 491, 210 (1997).

[15] T. J. Millar, C. Walsh, M. A. Cordiner, R. N. Chuimín, and E. Herbst, Astrophys. J. Lett. 662, L87 (2007).

[16] S. Harrison and J. Tennyson, J. Phys. B: At. Mol. Opt. Phys. 45, 035204 (2012).
[17] S. Harrison and J. Tennyson, J. Phys. B: At. Mol. Opt. Phys. 44, 045206 (2011).

[18] V. Kokoouline, N. Douguet, and C. H. Greene, Chem. Phys. Lett. 507, 1 (2011).

[19] D. A. Varshalovich, A. N. Moskalev, and V. K. Khersonskii, Quantum Theory of Angular Momentum (World Scientific, Singapore, 1988).

[20] H.-J. Werner, P. J. Knowles, R. Lindh, F. R. Manby, M. Schütz et al., MOLPRO, version 2008.3, a package of ab initio programs (2008).

[21] S. E. Bradforth, E. H. Kim, D. W. Arnold, and D. M. Neumark, J. Chem. Phys. 98, 800 (1993).

[22] D. Ajitha and K. Hirao, Chem. Phys. Lett. 347, 121 (2001).

[23] A. D. Pradhan, H. Partridge, and C. W. Bauschlicher, Jr., J. Chem. Phys. 101, 3857 (1994).

[24] K. P. Huber and G. Herzberg, Constants of Diatomic Molecules (Van Nostrand Reinhold, New York, 1979).

[25] C. W. McCurdy and T. N. Rescigno, Phys. Rev. A 39, 4487 (1989).

[26] A. E. Orel, T. N. Rescigno, and B. H. Lengsfield, Phys. Rev. A 44, 4328 (1991).

[27] D. Ajitah and K. Hirao, Phys. Lett. 347, 121 (2001).

[28] J. Thomson and F. W. Dalby, Can. J. Phys. 46, 2815 (1968).

[29] S. S. Kumar, D. Hauser, R. Jindra, T. Best, Š. Roučka, W. Geppert, T. J. Millar, and R. Wester, Astrophys. J. 776, 25 (2013).

[30] A. R. Hochstim, Kinetic Processes in Gases and Plasmas (Academic Press, New York, 1969).

[31] M. L. Senent and M. Hochlaf, Astrophys. J. 708, 1452 (2010).

[32] M. Ayouz, R. Lopes, M. Raoult, O. Dulieu, and V. Kokoouline, Phys. Rev. A 83, 052712 (2011).

[33] M. Aymar, C. H. Greene, and E. Luc-Koenig, Rev. Mod. Phys. 68, 1015 (1996). 